\title{
Risk factors and survival analysis of arrhythmia following lung cancer surgery: a retrospective study
}

\author{
Jialin Gong, Xiaofei Wang, Zuo Liu, Shuang Yao, Zengtuan Xiao, Mengzhe Zhang, Zhenfa Zhang \\ Department of Lung Cancer Surgery, Tianjin Medical University Cancer Institute and Hospital, Tianjin, China \\ Contributions: (I) Conception and design: J Gong, X Wang, Z Liu, Z Zhang; (II) Administrative support: J Gong, X Wang, Z Zhang; (III) Provision \\ of study materials or patients: Z Zhang; (IV) Collection and assembly of data: All authors; (V) Data analysis and interpretation: All authors; (VI) \\ Manuscript writing: All authors; (VII) Final approval of manuscript: All authors. \\ Correspondence to: Zhenfa Zhang, MD. Department of Lung Cancer Surgery, Tianjin Medical University Cancer Institute and Hospital, Huanhu \\ West Rd, Tianjin, China. Email: zhangzhenfa@tmu.edu.cn.
}

\begin{abstract}
Background: Surgical treatment of lung cancer is one of the important treatments for early-stage nonsmall cell lung cancer (NSCLC). However, arrhythmia, especially atrial fibrillation (AF) and supraventricular arrhythmia, are quite common among patients after surgical treatment of lung cancer. The impact of postoperative arrhythmia (PA) on survival is rarely reported. Our aim was to evaluate the risk factors of PA and its impact on overall survival (OS) after lung cancer surgery.

Methods: A total of 344 patients diagnosed with NSCLC who underwent lung cancer surgery were enrolled in this study. These patients were divided into two groups based on the occurrence of PA. Univariate and multivariate logistic regression analyses were conducted to identify the risk factors of PA. The Kaplan-Meier method was applied to show the OS differences between the two groups.

Results: The incidence of PA was 16\% (55/344). Among these 55 patients, 20 had AF, 30 had sinus tachycardia, and 5 had premature beats. A total of 332 patients underwent lung cancer radical resection. Operation type $(\mathrm{P}<0.001)$, preoperative abnormal ECG $(\mathrm{P}=0.032)$, transfusion $(\mathrm{P}=0.016)$, postoperative serum potassium concentration $(\mathrm{P}=0.001)$ and clinical stage $(\mathrm{P}<0.05)$ were risk factors for $\mathrm{PA}$. $\mathrm{PA}(\mathrm{HR} 2.083$, 95\% CI, 1.334-3.253; P=0.001), age (HR 1.543, 95\% CI, 1.063-2.239; P=0.025) and mediastinal lymph node metastasis (HR 2.655, 95\% CI, 1.809-3.897; $\mathrm{P}<0.001$ ) were independent prognostic risk factors for OS by multivariate cox analysis.

Conclusions: We identified PA as an independent prognostic risk factor to predict poor OS in patients who underwent lung cancer surgery and had risk factors for PA. We therefore provides guidance for PA in improving the prognosis of lung cancer patients.
\end{abstract}

Keywords: Lung cancer; surgery; postoperative arrhythmia (PA); prognosis

Submitted Aug 24, 2020. Accepted for publication Dec 11, 2020.

doi: $10.21037 /$ jtd-20-2740

View this article at: http://dx.doi.org/10.21037/jtd-20-2740

\section{Introduction}

Surgery is one of the most important treatments for earlystage non-small cell lung cancer (NSCLC). The procedure often leads to the removal of functional lung tissue, which may lead to hypoxemia and impair heart function. Most patients with lung cancer have a long history of smoking, which tends to damage the cardiopulmonary functions (1).
Therefore, arrhythmias commonly occur in patients after lung cancer surgery.

Postoperative arrhythmia (PA), especially postoperative atrial fibrillation (PAF), is a common postoperative complication in lung cancer patients. The prevalence of PAF is $10-15 \%$ after lobectomy and $20-30 \%$ after pneumonectomy (2). Although the PAF is usually transient, it is sometimes associated with other severe complications, 
including thromboembolic events, hemodynamic deterioration and heart failure (3). It has been reported that PAF could also extend the length of hospital stay and increase perioperative mortality (4). However, there have been few studies on whether arrhythmia has an influence on postoperative survival among patients with lung cancer.

Therefore, this study aimed to assess the impact of PA on the overall survival (OS) of patients after lung cancer resection and to identify risk factors associated with PA. We present the following article in accordance with the STROBE reporting checklist (available at http://dx.doi. org/10.21037/jtd-20-2740).

\section{Methods}

\section{Population and study design}

Between January 2007 and January 2008, we retrospectively reviewed 362 patients undergoing lung cancer surgery at Tianjin Medical University Cancer Hospital. Patients with complete preoperative examination information were included: a pulmonary function test, electrocardiogram (ECG), venous color Doppler ultrasound of the lower limbs, emission computed tomography, chest computed tomography and head computed tomography or magnetic resonance imaging; undergoing lung cancer surgery via thoracotomy or thoracoscope; and receiving a continuous telemetry strip after surgery. The following patients were excluded: patients with pace-maker devices, permanent $\mathrm{AF}$ and incomplete records. Finally, 344 patients were enrolled in this study. According to the occurrence of PA, these patients were divided into two groups: PA $(\mathrm{n}=55)$, and non-PA $(\mathrm{n}=289)$. The baseline data of these patients are shown in Table 1. The data were collected from the hospital medical records. The study has conformed to the provisions of the Declaration of Helsinki (as revised in 2013). This study was approved by the ethics committee of Tianjin Medical University Cancer Hospital (bc2020193) and individual consent was waived because of the retrospective nature of this study.

\section{The detection and definition of $P A$}

After surgery, all patients were given continuous ECG monitoring for at least 48 hours postoperatively. Once the telemetry showed arrhythmia disturbances or the patients had obvious symptoms such as palpitation and suffocation, a 12-lead ECG recording was obtained. The definition of $\mathrm{AF}$ was that the ECG recordings demonstrated the presence of characteristic ECG features of AF lasting for at least 30 seconds. Sinus tachycardia was defined as the ECG showing a sinus rhythm greater than 100 beats per minute and lasting more than 3 seconds, and premature beats were defined as more than 100 beats in 24 hours (both ventricular and atrial).

\section{Patient management and follow up}

After discharge, routine examinations such as a computed tomography scan of the chest, head, and abdomen and an ultrasound of the neck and abdomen were generally taken every 3 months for the first 2 years postoperatively and every 6 months for the next 5 years. Afterward, the patients were assessed annually. Follow-up data were collected by contacting the patients or their relatives via telephone or obtaining them from the hospital records. Eight patients were lost to contact after discharge in the PA group and six patients in the non-PA group. We compared the 14 patients with lost contact and the 330 patients who had followup information, and there was no statistically significant difference between the two groups $(\mathrm{P}>0.05$, Table $\mathrm{S} 1)$. The end-point of the study was OS, which was defined as the interval between the date of the operation and the date of death or censoring. The follow-up information and OS were both calculated in months. The follow-up was completed in December 2019 or to the date of the death of the patients.

\section{Statistical analysis}

Statistical analysis was performed using the Statistical Package for the Social Sciences version24.0 (SPSS Inc, Chicago). Categorical variables were compared using chisquare test or Fisher's exact test expressed as frequency and percentage. Continuous variables were tested for normal distribution by the Kolmogorov Smirnov test, then compared between groups with a t-test among the normal distribution variables and expressed as the mean \pm standard deviation. Those with skewed distributions were compared with the Mann-Whitney test and expressed as medians and interquartile range. Three groups of continuous variables in accordance with the normal distribution were compared by ANOVA test, and variables with skewed distributions were compared by the Kruskal-Wallis $\mathrm{H}$ test.

Univariate and multivariate logistic analyses were performed to predict the risk factors for PA. Survival was 
Table 1 Basic clinicopathologic features and perioperative data of patients

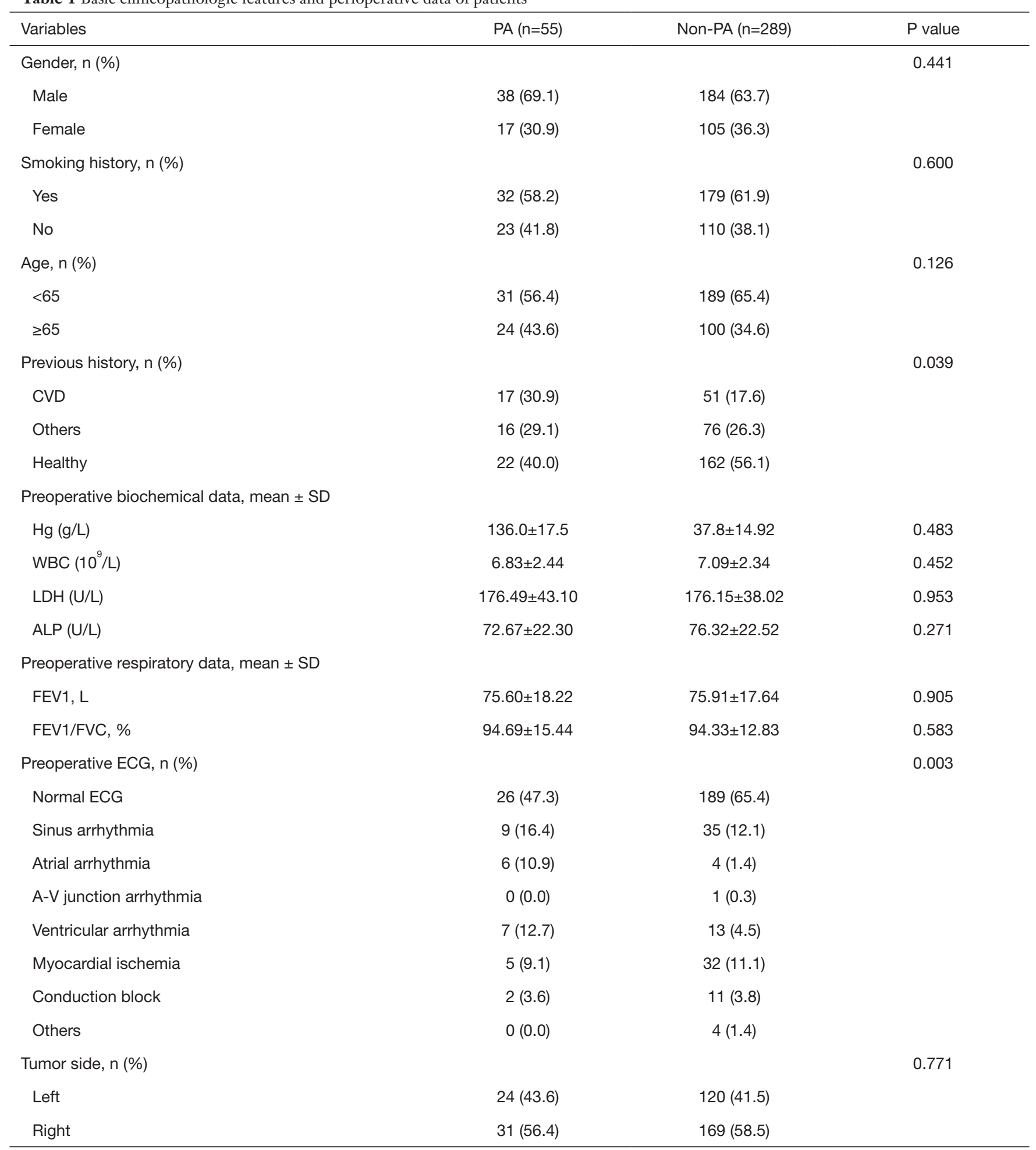

Table 1 (continued) 
Table 1 (continued)

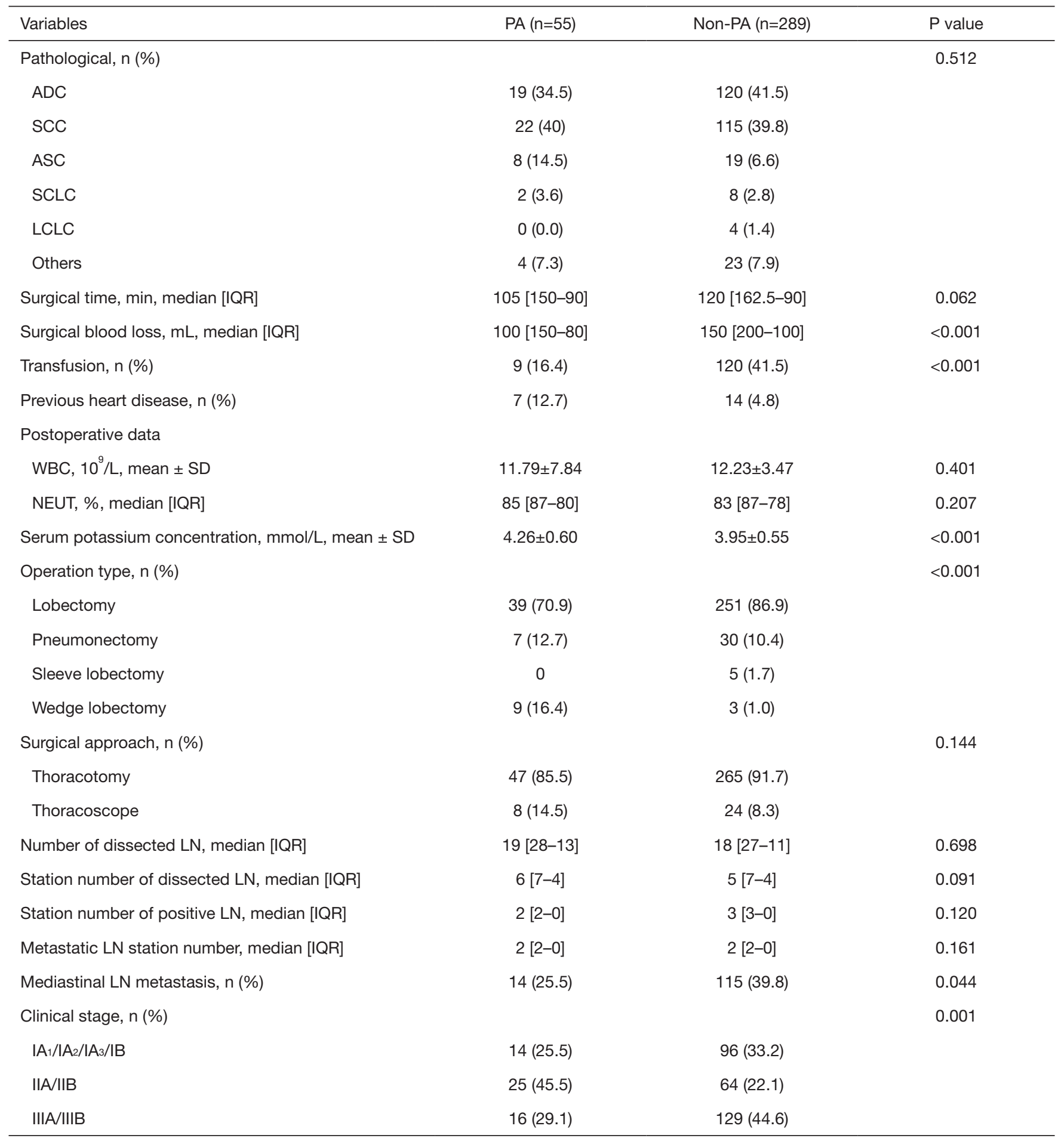

PA, postoperative arrhythmia; CVD, cardiovascular disease; SD, standard deviation; Hg, hemoglobin; WBC, white blood cell; LDH, lactate dehydrogenase; ALP, alkaline phosphatase; FEV1, forced expiratory ventilation in 1 second; FVC, forced vital capacity; ECG, electrocardiograph; A-V, atrioventricular; ADC, adenocarcinoma; SCC, squamous cell carcinoma; ASC, adenosquamous carcinoma; SCLC, small cell lung cancer; LCLC, large cell lung cancer; IQR, interquartile range; NEUT, neutrophil; LN, lymph node. 


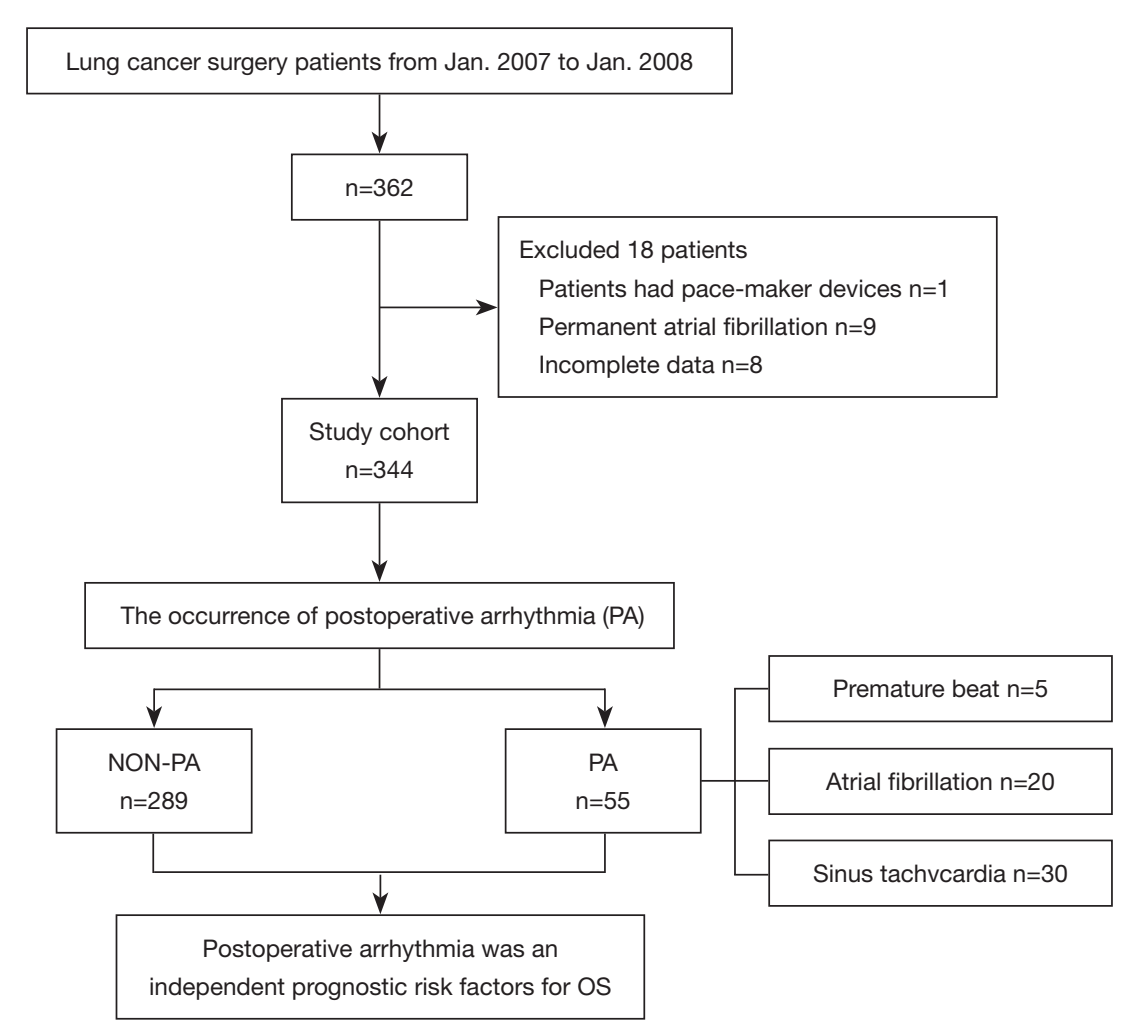

Figure 1 The flow chart.

estimated by the Kaplan-Meier method and compared using log-rank tests. Multivariate Cox proportional hazards regression analysis was used to calculate the hazard ratio (HR) and $95 \%$ CI. In all analyses, a two-tailed $\mathrm{P}<0.05$ was considered statistically significant.

\section{Results}

\section{Baseline data and operative characteristics}

With reference to the analysis of risk factors of PA in the previous literature (4-8), we collected the basic clinicopathologic features and perioperative data of these 344 patients as shown in Table 1, and the study process as shown in Figure 1. The results of the preoperative ECG showed significant difference between the patients in the PA and non-PA groups. Among the 55 patients, 26 (47.3\%) patients had normal ECG, 9 patients (16.4\%) had sinus arrhythmia, 6 patients $(10.9 \%)$ had an atrial arrhythmia, 7 patients $(12.7 \%)$ had ventricular arrhythmia, 5 patients $(9.1 \%)$ had myocardial ischemia and 2 patients (3.6\%) showed a conduction block. The results for their previous history was significantly different between two groups (all $\mathrm{P}<0.05$ ).
Among the 344 patients who underwent lung cancer surgery (thoracotomy and thoracoscopic surgery), the majority ( $\mathrm{n}=332,96.5 \%)$ underwent radical resection, including sleeve lobectomy $(\mathrm{n}=5,1.5 \%)$, lobectomy $(\mathrm{n}=290,84.3 \%)$ and pneumonectomy $(\mathrm{n}=37,10.5 \%)$, while nonradical resection consisted of a wedge lobectomy ( $\mathrm{n}=12$, $3.5 \%)$. Significant differences were seen between the patients with or without $\mathrm{PA}(\mathrm{P}<0.001)$. Moreover, different clinical stages also had significant differences in the PA group compared to the non-PA group, and 41 patients (74.5\%) were at least clinical stage II in the PA group. The median amount of blood loss of the PA group was 100 $\mathrm{mL}$, and that of the other group was $150 \mathrm{~mL}$. There was a statistically significant difference in blood loss amount between two groups $(\mathrm{P}<0.001)$.

\section{Characterization of $P A$}

Table 2 shows that the overall incidence of arrhythmia after lung cancer surgery was $16 \%$ (55/344), among which the most common arrhythmia type was sinus tachycardia $(\mathrm{n}=30)$, more common than $\mathrm{AF}(\mathrm{n}=20)$ and a premature beat 
Table 2 Baseline data of three postoperative arrythmias groups

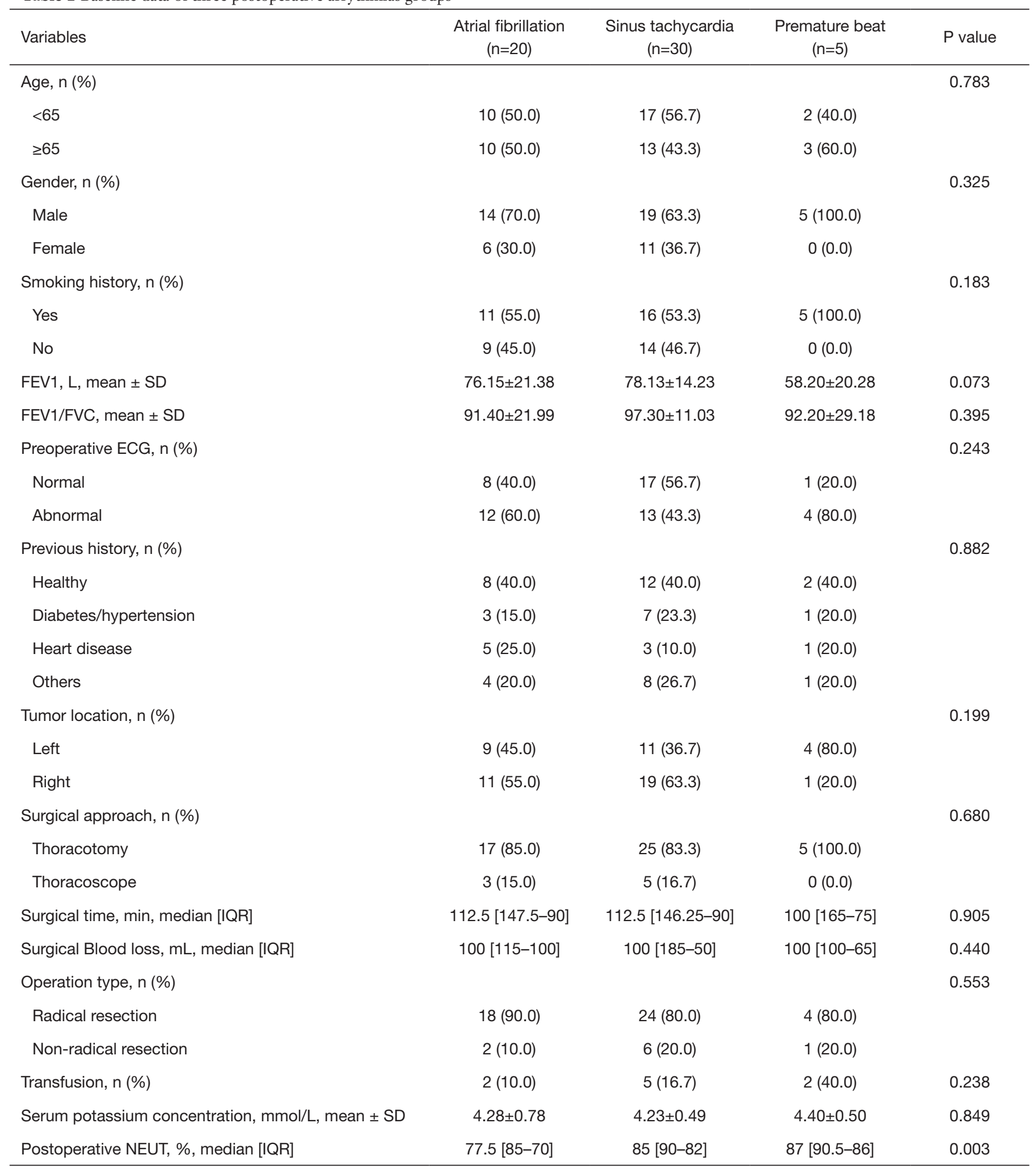

Table 2 (continued) 
Table 2 (continued)

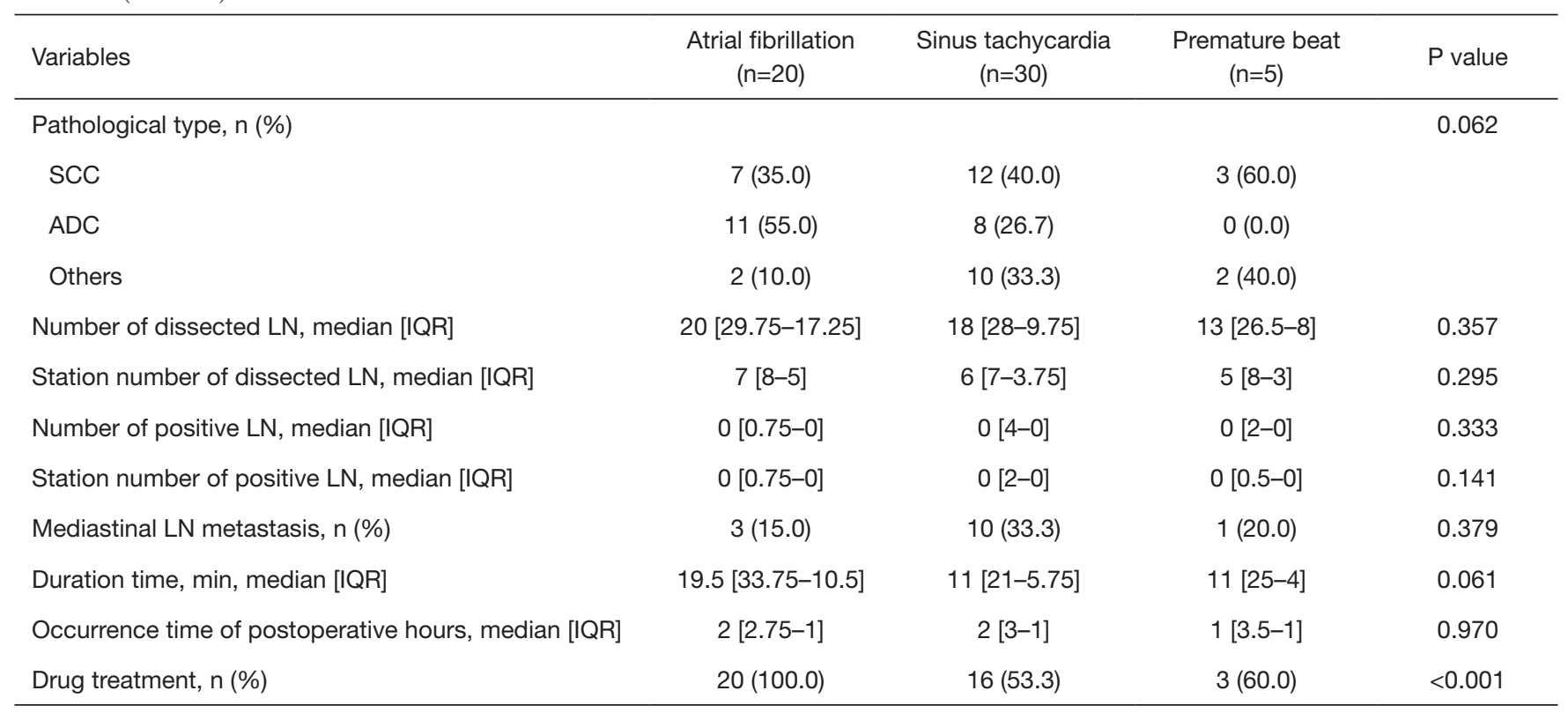

FEV1, forced expiratory ventilation in 1 second; FVC, forced vital capacity; ECG, electrocardiograph; SD, standard deviation; IRQ, interquartile range; NEUT, neutrophil; SCC, squamous cell carcinoma; ADC, adenocarcinoma; LN, lymph node.

$(\mathrm{n}=5)$. Among the 5 patients with premature beats, two patients were diagnosed with atrial premature beats and the other 3 patients were diagnosed with ventricular premature beats. These two patients with atrial premature beats and the two patients with ventricular premature beats were all bigeminy beats, while the one patient with ventricular premature beats had trigeminy beats. Among these patients, 16 had a transient arrhythmia and converted to a sinus rhythm without the need for medication, and the other 39 were given drugs to treat their arrhythmias $(\mathrm{P}<0.001$, Figure $2 A$ ). Thirty-nine patients were prescribed medications, and 11 were treated with digoxin $(20 \%), 9$ were treated with digoxin combined with verapamil (16.4\%), 6 were treated with digoxin plus metoprolol (10.9\%) and 3 were treat with furosemide $(5.5 \%)$. Three patients with premature beats were treated with lidocaine and moricizine to control their ventricular rate. PA usually occurred on the first day after surgery, and it peaked in the first hours after surgery. It lasted from 1 to 72 hours, and the average duration of each type of arrhythmia is shown in Figure $2 B$.

\section{Univariate and multivariate logistic analyses to identify risk factors of $P A$}

The results of the univariate and multivariate logistic analyses are shown in Table 3. Univariate regression revealed that a history of heart disease (coronary heart disease, arrhythmia, etc.), preoperative abnormal ECG performance, transfusion, mediastinal lymph node metastasis, postoperative serum potassium concentration, operation type and the clinical stage were significant factors to predict PA. The blood loss amount was also statistically significant.

The variables with $\mathrm{P}<0.05$ in univariate analysis were included in the multivariate analysis using a forward method. The results of the multivariate logistic regression showed that preoperative abnormal ECG, postoperative serum potassium concentration, transfusion, operation type and clinical stage were independent factors for the development of PA. The OR for preoperative abnormal ECG was 2.167 ( $\mathrm{P}=0.032,95 \%$ CI, 1.070-4.390) compared with patients with a preoperative normal ECG. The ORs for postoperative serum potassium concentration and transfusion were $2.706(\mathrm{P}=0.001,95 \% \mathrm{CI}, 1.467-4.991)$ and $2.798(\mathrm{P}=0.016,95 \% \mathrm{CI}, 1.208-6.480)$ respectively. The OR of patients undergoing lung cancer radical dissection was $15.687(\mathrm{P}<0.001,95 \% \mathrm{CI}, 3.665-67.136)$ compared with patients undergoing wedge lobectomy (nonradical dissection). The ORs of stage II patients were $3.060(\mathrm{P}=0.015,95 \% \mathrm{CI}, 1.247-7.507)$ compared with 

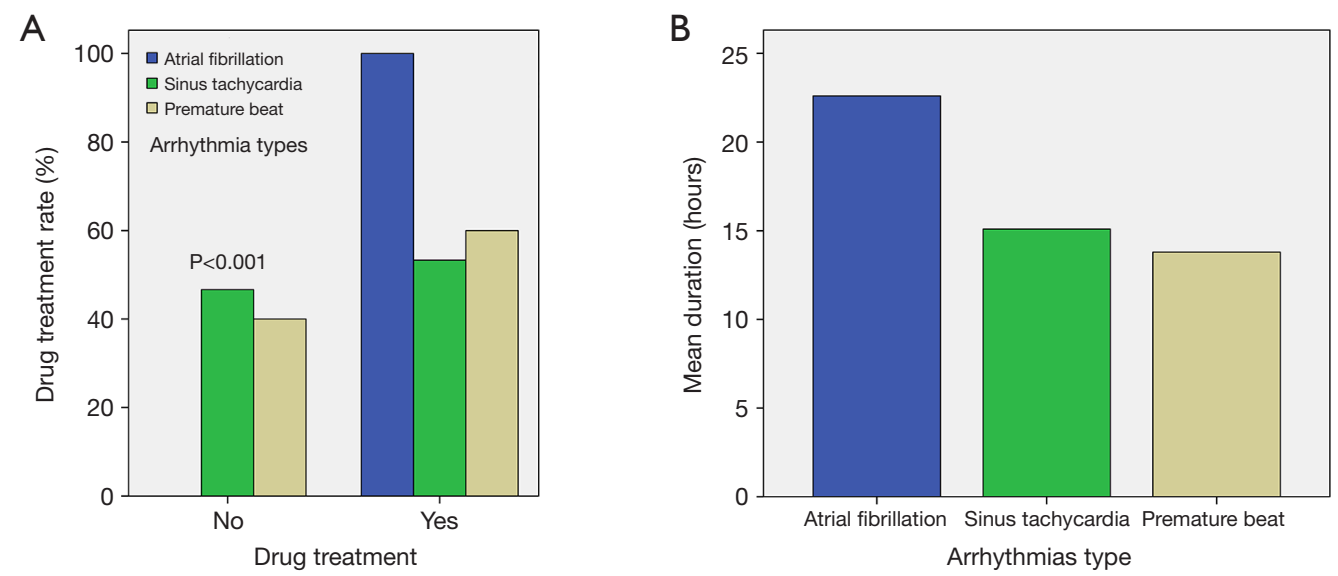

Figure 2 The drug treatment rate of each kind of postoperative arrhythmia (A) and the mean duration of each type of arrhythmia (B).

stage I or III.

\section{Long term survival and analysis of survival factors}

As Figure 3 shows, the five-year and ten-year survival rates in patients with $\mathrm{PA}$ were $39.7 \%$ and $33.6 \%$ respectively and that in patients without PA were $57.1 \%$ and $56.5 \%$ respectively. Log rank test showed that $\mathrm{PA}(\mathrm{P}=0.006)$, clinical stage $(\mathrm{P}<0.001)$, TNM classification $(\mathrm{P}=0.002)$, the number of dissected lymph node $(\mathrm{P}<0.001)$, the number of metastatic lymph node stations $(\mathrm{P}<0.001)$, mediastinal lymph node metastasis $(\mathrm{P}<0.001)$ and the number of $\mathrm{N} 2$ metastatic stations $(\mathrm{P}<0.001)$ were associated with a poor OS.

The duration of follow-up after surgery was 155 months in the study population. The median follow-up duration was 26 months in the PA group and it was 41 months in the other group. The cause of death was lung cancer in most cases. A total of 135 patients died during the follow-up period. Twenty-six patients died in the PA group and 106 patients without PA died. Among these 106 patients who died without $\mathrm{PA}$, one patient died due to a pulmonary infection in the hospital and the other 105 patients died due to lung cancer recurrence or metastasis. Among these 26 patients who died with PA, 2 patients died after surgery in the hospital and 4 patients died of heart disease in the follow-up period, while the rest died of cancer recurrence or metastasis.

The results of the Cox proportional hazards analyses are shown in Table 4. Age, clinical stage, mediastinal lymph node metastasis, the number of positive lymph nodes, the station number of the positive lymph nodes, the station number of the positive $\mathrm{N} 2$ lymph nodes and PA were all significant factors by univariate analysis (all $\mathrm{P}<0.05)$. PA
(HR 1.995, 95\% CI, 1.261-3.157; $\mathrm{P}=0.003$ ), age (HR 1.543, 95\% CI, 1.063-2.239; $\mathrm{P}=0.023)$ and mediastinal lymph node metastasis (HR 2.657, 95\% CI, 1.802-3.918; $\mathrm{P}<0.001$ ) were identified as independent prognostic risk factors by multivariate analysis.

\section{Discussion}

Arrythmia, especially AF, is the most common cardiac complication following lung cancer surgery. According to a previous review (9), its incidence ranges from $10 \%$ to $20 \%$ after lobectomy and up to $40 \%$ after pneumonectomy (more common in the right side). Our study revealed similar observations: patients who had right-sided surgery were more likely to experience PA $(n=31)$ than patients who had left-sided surgery $(\mathrm{n}=24)$.

New-onset AF usually occurs within a week after the lung cancer surgery, and peaks in 2 to 4 days (10). Ivanovic et al. (6) reported that $\mathrm{AF}$ always occurred in the first 24 hours after the operation, while the average time of arrhythmia appearing in our study was earlier than in these previously reported data. The shorter time before the appearance of an arrhythmia after surgery may be related to the fact that the patients were admitted directly to the thoracic intensive care unit after surgery and received continuous ECG monitoring.

The reasons for PA are complex and not fully understood. There is evidence showing a strong correlation between PA and pulmonary vein as well as other atrial sites (11), but a specific mechanism has yet to be found. For instance, inappropriate intraoperative management of the pulmonary vein may trigger PA (12), while postoperative 
Table 3 Results of univariate and multivariate analyses to identify risk factors of postoperative arrhythmia

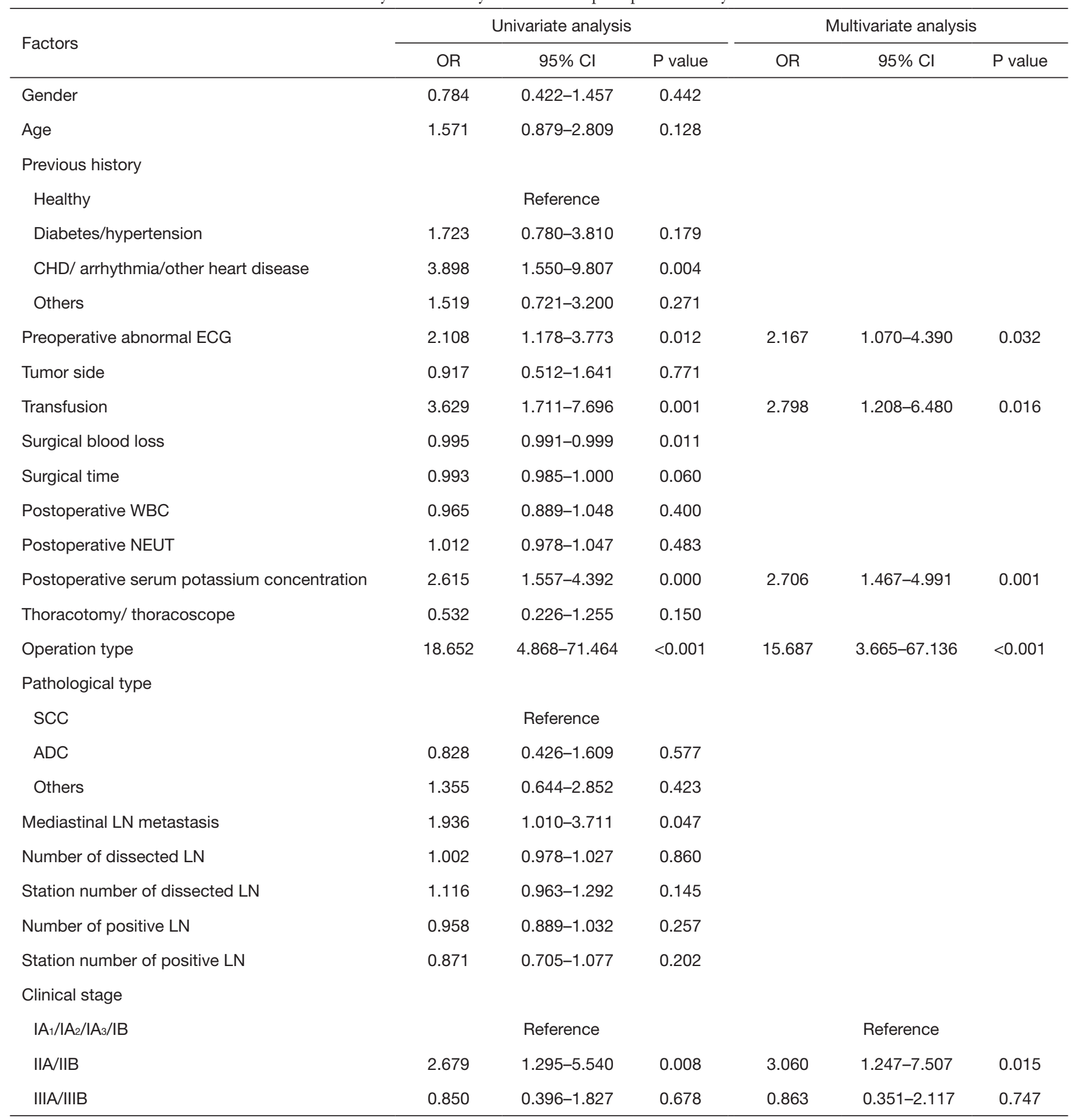

CHD, coronary heart disease; ECG, electrocardiogram; WBC, white blood cell; NEUT, neutrophil; SCC, squamous cell carcinoma; ADC, adenocarcinoma; LN, lymph node. 


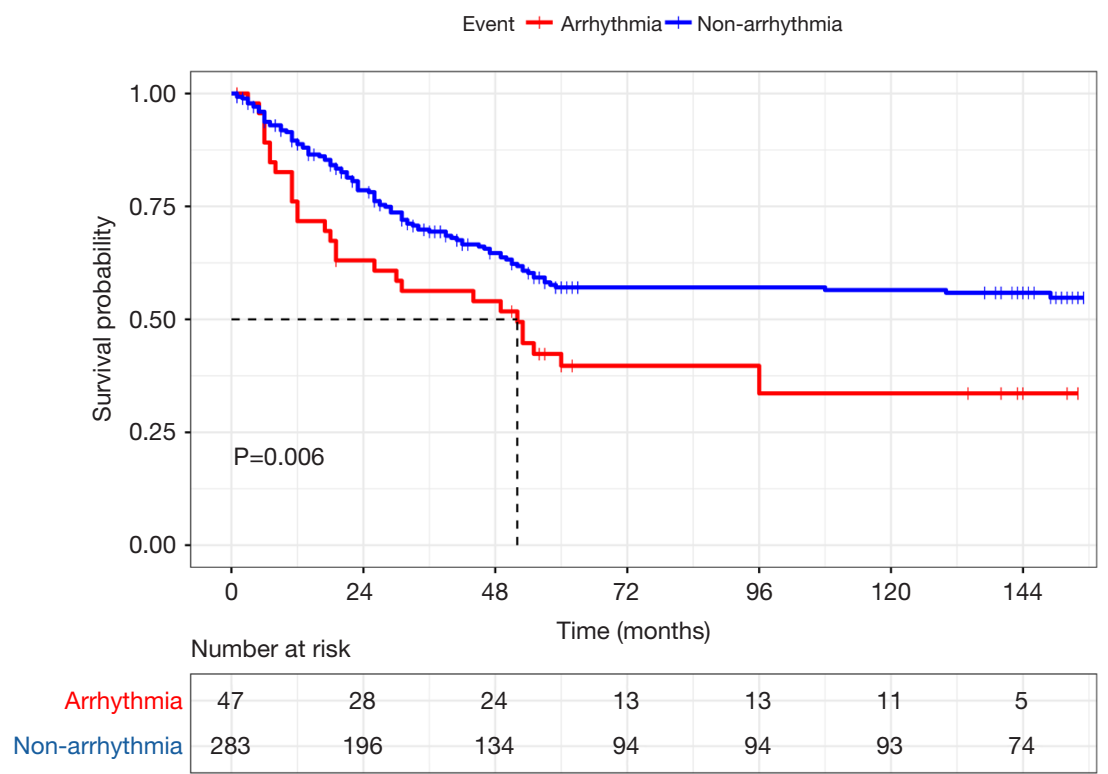

Figure 3 Kaplan-Meier curves for patients with postoperative arrhythmia and patients without postoperative arrhythmia.

pain could trigger a sympathetic response that contributes to the PA (13). Pain-induced sympathetic outflow and the imbalance between the sympathetic and parasympathetic activity could produce atrial ectopic beats and, in susceptible patients, trigger PAF (14).

The 2012 National Comprehensive Cancer Network (NCCN) Lung Cancer Guidelines recommended thoracoscopic lobectomy as a radical surgical procedure for early NSCLC (15). A recent guideline divided the surgical procedure into three different risk levels based on the likelihood of PAF, with lobectomy and pneumonectomy placed in the high risk group ( $>15 \%$ incidence) (16). Radical resection, especially pneumonectomy $(4,9)$, is a strong independent risk factor for PA. The operation process decreases the pulmonary tissue and capillary bed. Pulmonary capillary bed reduction and insufficiency of pulmonary ventilation function after lung resection would result in a decline of the lung ventilation/perfusion ratio and an increase in the pulmonary capillary blood flow (1). During the surgery process, mechanical manipulation of the heart and pericardium leads to local tissue trauma and local inflammatory responses which may both lead to PAF (17-19). An analysis of the Society of Thoracic Surgeons database also demonstrated that increasing the extent of the operations was another risk factor for postoperative $\mathrm{AF}$ (20), and De Decker et al. (9) reported that the volume of pulmonary resection was a critical risk factor for the development of PAF.

The identified risk factors for the development of sustained postoperative AF are almost identical to those known to make the atrium vulnerable to the development of $\mathrm{AF}$ in the nonsurgical setting (16). Several risk factors have been identified for the development of PAF, such as age, male gender, heart disease history and higher clinical stages $(4-8,20)$. In the current study, a preoperative abnormal ECG, operation type, higher clinical stages, postoperative serum potassium concentration and the need for transfusion were independently associated with PA development in multivariate analysis.

A Japanese group has reported that the surgical approach itself is another risk factor for PA (8). However, their results contradict the findings of the current study, and one possible explanation could be that the sample size of the thoracotomy surgery patients $(n=312)$ was much larger than that of the thoracoscopy patients $(n=32)$. Previous studies reported that mediastinal lymph node dissection was considered to be a risk factor for postoperative $\mathrm{AF}(8,21)$. The reason for mediastinal lymph node metastasis being correlated with PA may be surgical management during mediastinal lymph node dissection, which is carried out near the vagal nerve and its branches, especially the cardiac plexus and pulmonary veins (22). This fits into the purported origin of paroxysmal $\mathrm{AF}$ in the pulmonary veins (11).

In the current study, the postoperative serum potassium 
Table 4 Univariate and multivariate cox predictors to postoperative arrythmia mortality

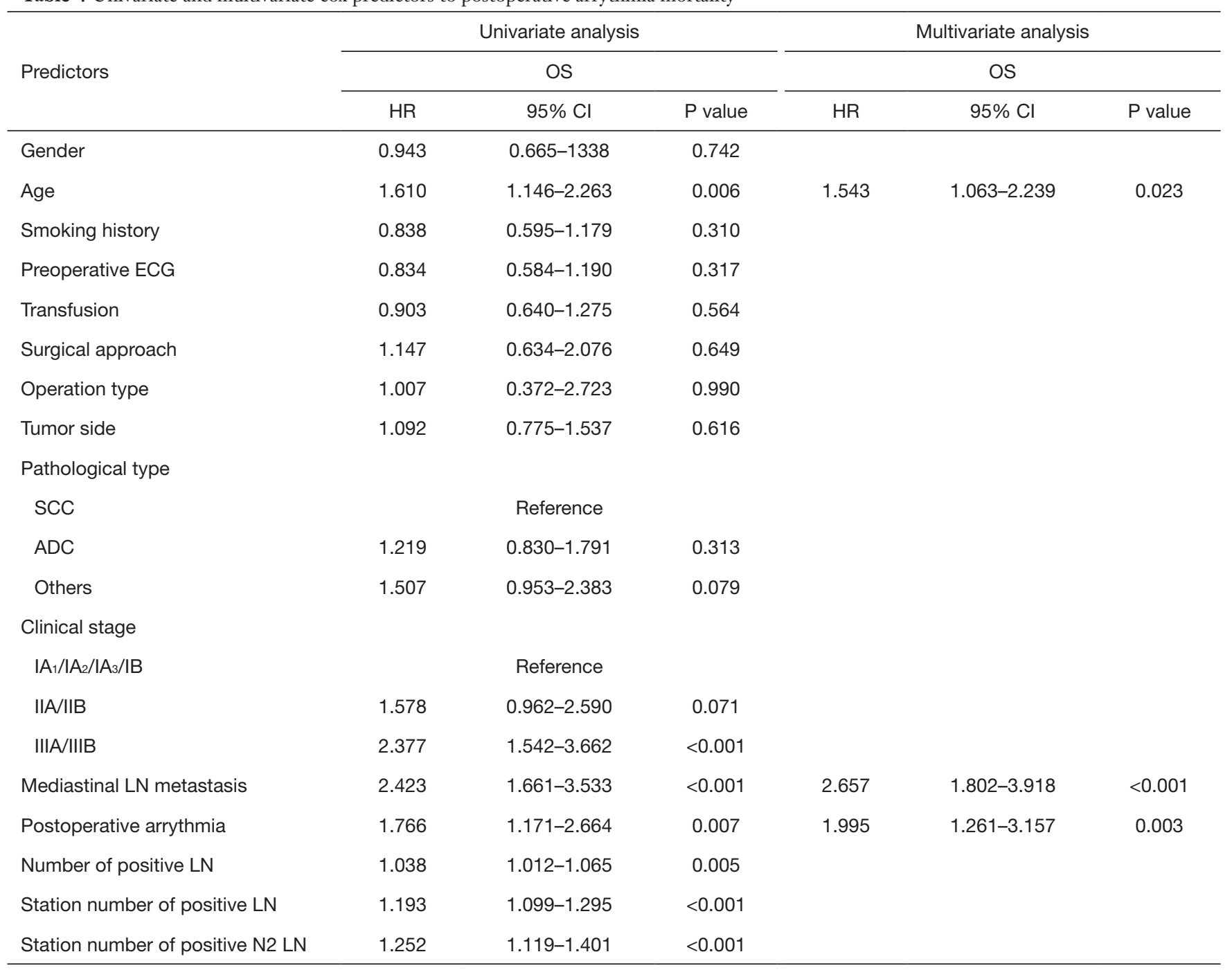

ECG, electrocardiograph; SCC, squamous cell carcinoma; ADC, adenocarcinoma; LN, lymph node.

concentration (OR 2.706, 95\% CI, 1.467-4.991, $\mathrm{P}=0.001$ ) and the need for a transfusion after surgery (OR 2.798, 95\% CI, 1.208-6.480, $\mathrm{P}=0.016)$ were statistically significant in multivariate analysis to predict PA. Electrolyte disturbance has been proven to cause postoperative supraventricular arrhythmias (23). A tight preoperative control of electrolytes is recommended to decrease the incidence of PAF (24). Walsh et al. (25) reported that in a prospective study, an arrhythmia after colorectal surgery may be caused by a low intracellular potassium concentration. Potassium deficiency may precipitate arrhythmias (13). The need for a transfusion usually means hypovolemia and it is likely that transfusion acts as a marker of a more complex or difficult dissection (21). It has been demonstrated that intraoperative hypovolemia, together with hypoxia and anemia, may lead to ischemia of atrial cells and myocardial conduction tissue, altering the cell's electric properties and leading to arrhythmias, both ventricular and supraventricular (26-28).

One of the major findings of the current study was that patients with PA had a poor prognosis compared to patients without it. The Cox proportional hazards regression analysis showed that $\mathrm{PA}$ was an independent risk factor for the prognosis after lung cancer surgery. However, the effects of PA on survival after lung cancer surgery are controversial. Cardinale et al. (29) reported that postoperative $\mathrm{AF}$ did not have any negative impact on 
short- and long-term mortality or on the recurrence rate in a prospective study of 233 people with 3-year follow-up. Amar et al. (30) reported that supraventricular tachycardia dysrhythmias occurrence after resection of NSCLC is associated with OS, while Imperatori et al. (5) reported that AF after pulmonary lobectomy for lung cancer, in addition to causing higher hospital morbidity and mortality, predicted a worse outcome in 5-year survivors. With a median follow-up period of 6 months, the recorded 1-, 5-, 10 -year OS rate were $98 \%, 42 \%$, and $31 \%$ respectively among patients with PAF. This result indicated that PA was an independent risk factor for OS, but no significant factor was found in the arrhythmia subgroup.

The risk factors of PA are complex and diverse and are closely related to the operation type, preoperative ECG, clinical stage, transfusion and postoperative potassium concentration. The occurrence of PA is associated with a poor prognosis, and therefore, it is necessary to actively treat basic heart diseases preoperatively and detect electrolyte concentrations. The selection of surgical treatment options should be individualized to reduce the surgical trauma, and postoperative management should be strengthened, such as a prolonged ECG monitoring time and reducing postoperative pain and other stimuli. It should be timely corrected to shorten the duration of arrhythmia and to reduce the adverse consequences caused by arrhythmias.

This study has several limitations. First, B-type natriuretic peptide (BNP) is an effective risk predictor for new-onset AF after lung cancer surgery (31), as well as the ultrasonic cardiogram results (32). However, these factors were not included in the current study, and the results suggest that patients with abnormal perioperative ECG should be tested for BNP and undergo echocardiography. Second, this is a single-center retrospective study, and the sample size is limited. Moreover, the follow-up information does not include the recurrence $\mathrm{AF}$ and other arrythmias, and the relationship among these variables and recurrent arrythmia have also has not been evaluated. Finally, there were many patients who lost contact with the research team after surgery. Those patients also ceased to participate in regular examinations at the clinic. Therefore, there is a need for prospective, multicenter and larger-scale trials in order to study the effects of arrhythmias on OS after lung cancer surgery.

\section{Conclusions}

In conclusion, we found that a preoperative abnormal ECG, transfusion, postoperative serum potassium, clinical stage and operation type were independent risk factors of PA. We identified $\mathrm{PA}$ as an independent prognostic risk factor to predict a poor OS in patients who underwent lung cancer surgery. This, therefore, provides guidance for evaluating PA to improv the prognosis of lung cancer patients.

\section{Acknowledgments}

Funding: This work was funded by the National Natural Science Foundation of China [Grant number 81772488].

\section{Footnote}

Reporting Checklist: The authors have completed the STROBE reporting checklist. Available at http://dx.doi. org/10.21037/jtd-20-2740

Data Sharing Statement: Available at http://dx.doi. org/10.21037/jtd-20-2740

Peer Review File: Available at http://dx.doi.org/10.21037/jtd20-2740

Conflicts of Interest: All authors have completed the ICMJE uniform disclosure form (available at http://dx.doi. org/10.21037/jtd-20-2740). The authors have no conflicts of interest to declare.

Ethical Statement: The authors are accountable for all aspects of the work in ensuring that questions related to the accuracy or integrity of any part of the work are appropriately investigated and resolved. The study has conformed to the provisions of the Declaration of Helsinki (as revised in 2013). This study was approved by the ethics committee of Tianjin Medical University Cancer Hospital (bc2020193) and individual consent was waived because of the retrospective nature of this study.

Open Access Statement: This is an Open Access article distributed in accordance with the Creative Commons Attribution-NonCommercial-NoDerivs 4.0 International License (CC BY-NC-ND 4.0), which permits the noncommercial replication and distribution of the article with the strict proviso that no changes or edits are made and the original work is properly cited (including links to both the formal publication through the relevant DOI and the license). See: https://creativecommons.org/licenses/by-nc- 
nd/4.0/.

\section{References}

1. Izquierdo JM, Pac JJ, Casanova J, et al. Lung resection surgery in patients with functional limits. Arch Bronconeumol 1995;31:328-32.

2. Shrivastava V, Nyawo B, Dunning J, et al. Is there a role for prophylaxis against atrial fibrillation for patients undergoing lung surgery? Interact Cardiovasc Thorac Surg 2004;3:656-62.

3. Guha A, Dey AK, Jneid H, et al. Atrial Fibrillation in the Era of Emerging Cancer Therapies. Eur Heart J 2019;40:3007-10.

4. Roselli EE, Murthy SC, Rice TW, et al. Atrial fibrillation complicating lung cancer resection. J Thorac Cardiovasc Surg 2005;130:438-44.

5. Imperatori A, Mariscalco G, Riganti G, et al. Atrial fibrillation after pulmonary lobectomy for lung cancer affects long-term survival in a prospective single-center study. J Cardiothorac Surg 2012;7:4.

6. Ivanovic J, Maziak DE, Ramzan S, et al. Incidence, severity and perioperative risk factors for atrial fibrillation following pulmonary resection. Interact Cardiovasc Thorac Surg 2014;18:340-6.

7. Kanzaki R, Kimura T, Kawamura T, et al. Outcomes of surgery for lung cancer in patients with atrial fibrillation as a preoperative comorbidity: a decade of experience at a single institution in Japan. Surg Today 2017;47:795-801.

8. Muranishi Y, Sonobe M, Menju T, et al. Atrial fibrillation after lung cancer surgery: incidence, severity, and risk factors. Surg Today 2017;47:252-8.

9. De Decker K, Jorens PG, Van Schil P. Cardiac complications after noncardiac thoracic surgery: an evidence-based current review. Ann Thorac Surg 2003;75:1340-8.

10. Lee G, Wu H, Kalman JM, et al. Atrial fibrillation following lung transplantation: double but not single lung transplant is associated with long-term freedom from paroxysmal atrial fibrillation. Eur Heart J 2010;31:2774-82.

11. Haïssaguerre M, Jaïs $P$, Shah DC, et al. Spontaneous initiation of atrial fibrillation by ectopic beats originating in the pulmonary veins. N Engl J Med 1998;339:659-66.

12. Hogue CW Jr, Creswell LL, Gutterman DD, et al. Epidemiology, mechanisms, and risks: American College of Chest Physicians guidelines for the prevention and management of postoperative atrial fibrillation after cardiac surgery. Chest 2005;128:9S-16S.
13. Chelazzi C, Villa G, De Gaudio AR. Postoperative atrial fibrillation. ISRN Cardiol 2011;2011:203179.

14. Jiang Z, Dai JQ, Shi C, et al. Influence of patientcontrolled i.v. analgesia with opioids on supraventricular arrhythmias after pulmonary resection. Br J Anaesth 2009;103:364-8.

15. Ettinger DS, Akerley W, Borghaei H, et al. Non-small cell lung cancer. J Natl Compr Canc Netw 2012;10:1236-71.

16. Frendl G, Sodickson AC, Chung MK, et al. 2014 AATS guidelines for the prevention and management of perioperative atrial fibrillation and flutter for thoracic surgical procedures. J Thorac Cardiovasc Surg 2014;148:e153-93.

17. Oka T, Ozawa Y, Ohkubo Y. Thoracic epidural bupivacaine attenuates supraventricular tachyarrhythmias after pulmonary resection. Anesth Analg 2001;93:253-9, 1st contents page.

18. Ahn HJ, Sim WS, Shim YM, et al. Thoracic epidural anesthesia does not improve the incidence of arrhythmias after transthoracic esophagectomy. Eur J Cardiothorac Surg 2005;28:19-21.

19. Liu L, Nattel S. Differing sympathetic and vagal effects on atrial fibrillation in dogs: role of refractoriness heterogeneity. Am J Physiol 1997;273:H805-16.

20. Onaitis M, D'Amico T, Zhao Y, et al. Risk factors for atrial fibrillation after lung cancer surgery: analysis of the Society of Thoracic Surgeons general thoracic surgery database. Ann Thorac Surg 2010;90:368-74.

21. Vaporciyan AA, Correa AM, Rice DC, et al. Risk factors associated with atrial fibrillation after noncardiac thoracic surgery: analysis of 2588 patients. J Thorac Cardiovasc Surg 2004;127:779-86.

22. Wu DH, Xu MY, Mao T, et al. Risk factors for intraoperative atrial fibrillation: a retrospective analysis of 10,563 lung operations in a single center. Ann Thorac Surg 2012;94:193-7.

23. Goldman L. Supraventricular tachyarrhythmias in hospitalized adults after surgery. Clinical correlates in patients over 40 years of age after major noncardiac surgery. Chest 1978;73:450-4.

24. Walsh SR, Tang T, Wijewardena C, et al. Postoperative arrhythmias in general surgical patients. Ann R Coll Surg Engl 2007;89:91-5.

25. Walsh SR, Oates JE, Anderson JA, et al. Postoperative arrhythmias in colorectal surgical patients: incidence and clinical correlates. Colorectal Dis 2006;8:212-6.

26. von Knorring J, Lepäntalo M, Lindgren L, et al. Cardiac arrhythmias and myocardial ischemia after thoracotomy 
for lung cancer. Ann Thorac Surg 1992;53:642-7.

27. Shields TW, Ujiki GT. Digitalization for prevention of arrhythmias following pulmonary surgery. Surg Gynecol Obstet 1968;126:743-6.

28. Sørensen O, Waaben J, Andersen KB, et al. The incidence of cardiac arrhythmias and arterial hypotension subsequent to standardized surgical stimuli in patients undergoing thoracotomy. With reference to enflurane and halothane. Acta Anaesthesiol Scand 1986;30:630-2.

29. Cardinale D, Martinoni A, Cipolla CM, et al. Atrial fibrillation after operation for lung cancer: clinical and prognostic significance. Ann Thorac Surg 1999;68:1827-31.
30. Amar D, Burt M, Reinsel RA, et al. Relationship of early postoperative dysrhythmias and long-term outcome after resection of non-small cell lung cancer. Chest 1996;110:437-9.

31. Nojiri T, Inoue M, Shintani Y, et al. B-type natriuretic peptide-guided risk assessment for postoperative complications in lung cancer surgery. World J Surg 2015;39:1092-8.

32. Al-Omari MA, Finstuen J, Appleton CP, et al. Echocardiographic assessment of left ventricular diastolic function and filling pressure in atrial fibrillation. Am J Cardiol 2008;101:1759-65.
Cite this article as: Gong $\mathrm{J}$, Wang $\mathrm{X}$, Liu Z, Yao S, Xiao Z, Zhang M, Zhang Z. Risk factors and survival analysis of arrhythmia following lung cancer surgery: a retrospective study. J Thorac Dis 2021;13(2):847-860. doi: 10.21037/jtd-20-2740 


\section{Supplementary}

Table S1 Basic data of lost contact patients and not lost contact patients

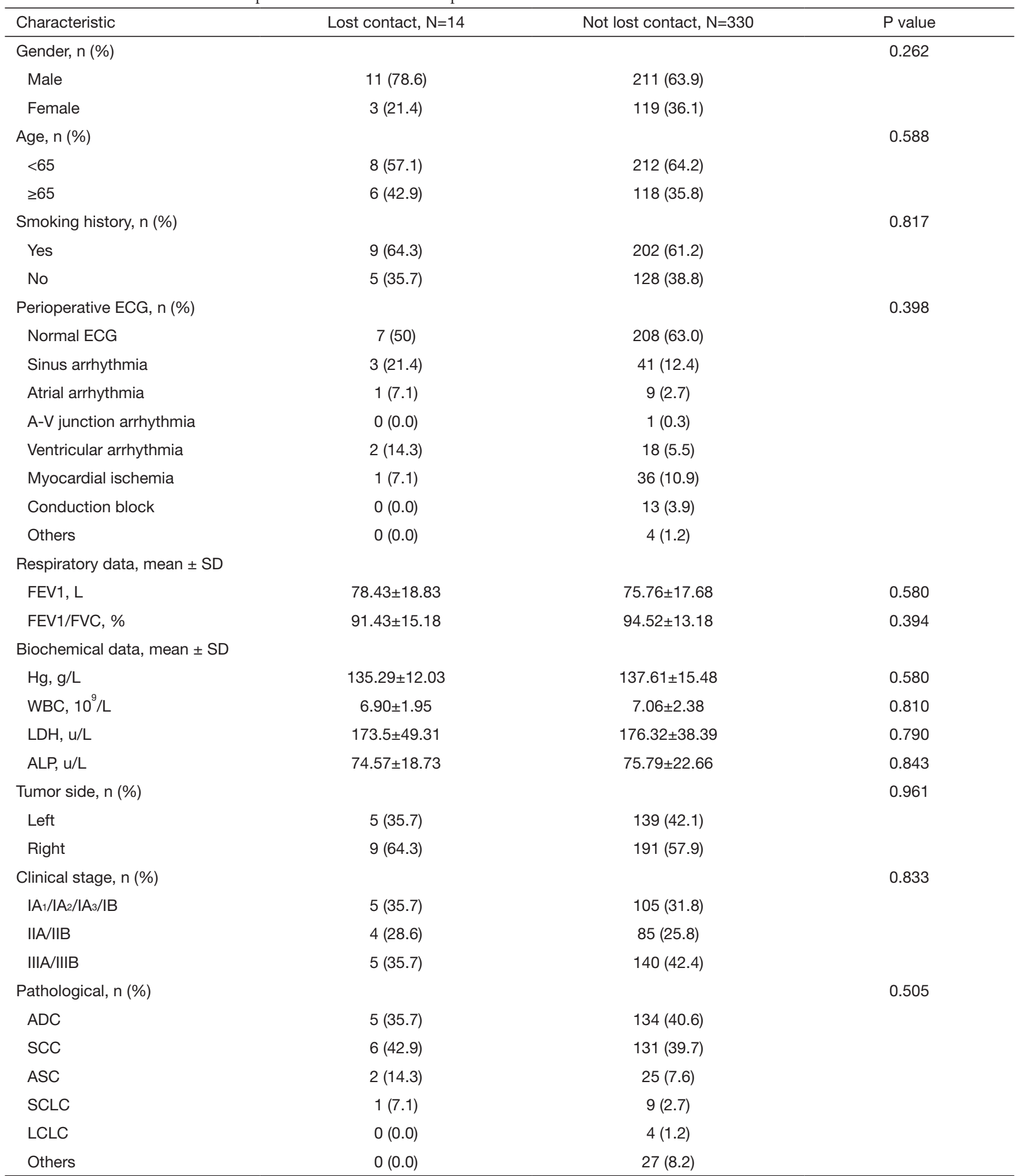

ECG, electrocardiograph; A-V, atrioventricular; SD, standard deviation; FEV1, forced expiratory ventilation in 1 second; FVC, forced vital capacity; Hg, hemoglobin; WBC, white blood cell; LDH, lactate dehydrogenase; ALP, alkaline phosphatase; ADC, adenocarcinoma; SCC, squamous cell carcinoma; ASC, adenosquamous carcinoma; SCLC, small cell lung cancer; LCLC, large cell lung cancer. 